Universidad de Lima

Facultad de Psicología

Carrera de Psicología

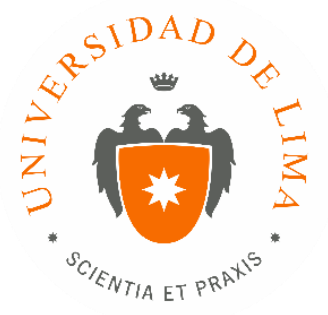

\title{
ORIENTACIÓN PRE VOCACIONAL EN UNA INSTITUCIÓN EDUCATIVA PÚBLICA
}

Trabajo de suficiencia profesional para optar el título profesional de Licenciado en Psicología

\section{Daniela Sotomayor Harm}

Código 20080985

$$
\text { Lima - Perú }
$$

Febrero de 2019 


\section{ORIENTACIÓN PRE VOCACIONAL EN UNA INSTITUCIÓN EDUCATIVA PÚBLICA \\ ÁREA EDUCATIVA}




\section{TABLA DE CONTENIDO}

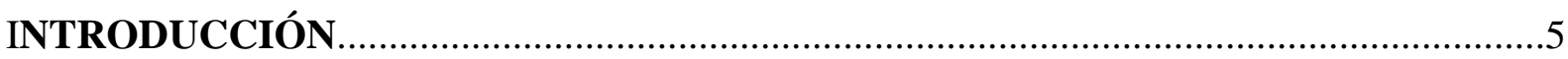

CAPÍTULO I: IDENTIFICACIÓN DEL PROBLEMA ................................................6

CAPÍTULO II: DESCRIPCIÓN DE LAS ACTIVIDADES REALIZADAS ...................9

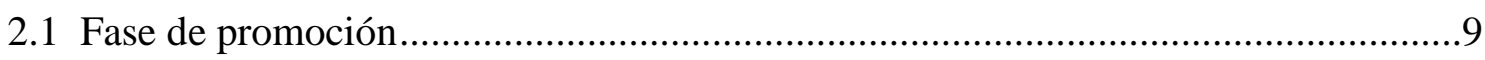

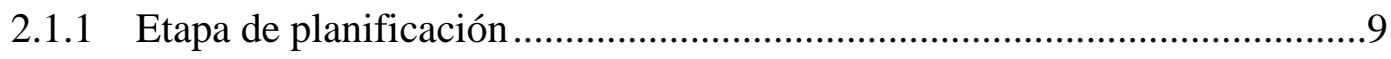

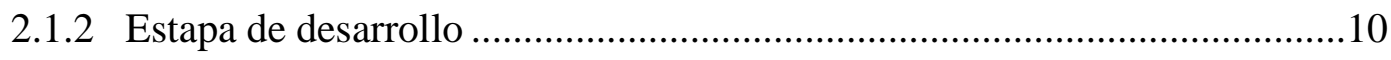

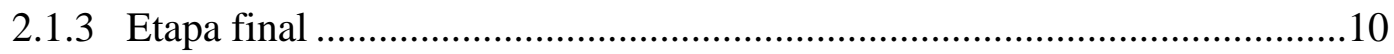

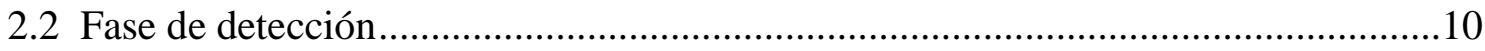

2.2.1 Etapa de planificación ..........................................................................10

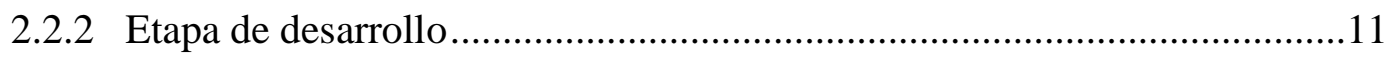

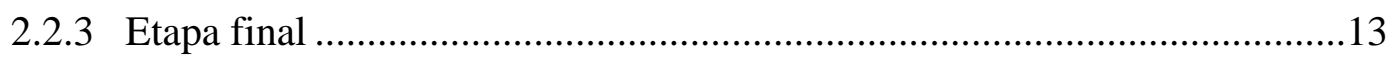

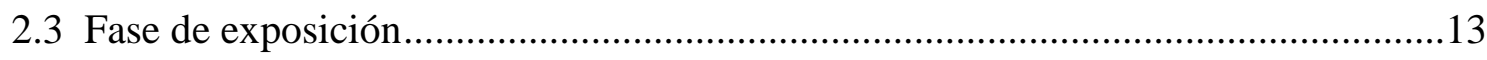

2.3.1 Etapa de planificación .................................................................................13

2.3.2 Etapa de desarrollo...............................................................................

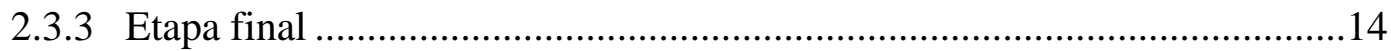

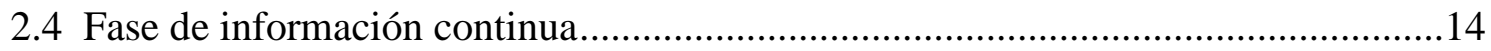

CAPÍTULO III: RESULTADOS DE LA INTERVENCIÓN ........................................15

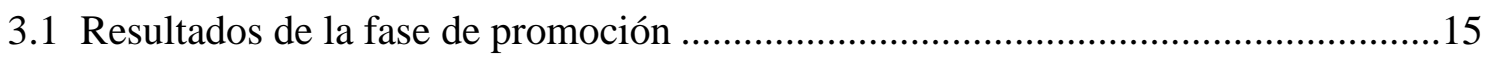

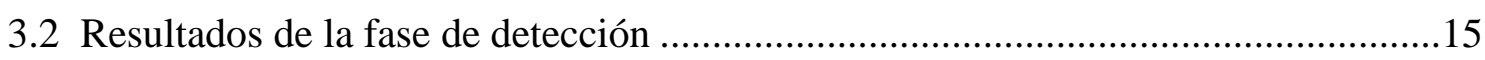

3.3 Resultados de la fase de exposición .................................................................. 16

3.4 Resultados de la fase de información continua ...................................................17

3.5 Resultados del Programa de Orientación Pre Vocacional ........................................17

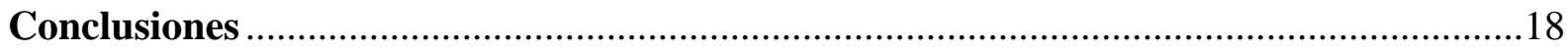

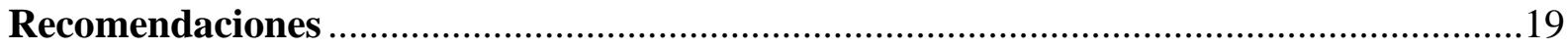

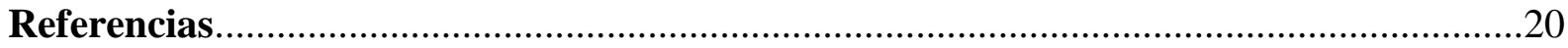

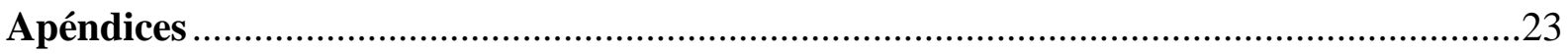




\section{ÍNDICE DE APÉNDICES}

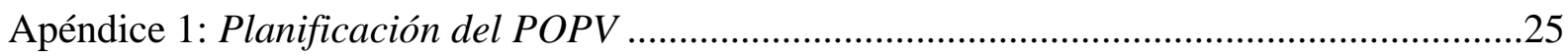

Apéndice 2: Resumen de las tareas realizadas.....................................................................26

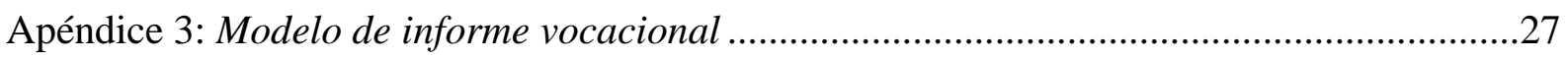

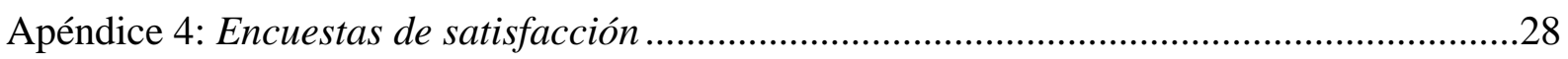

Apéndice 5: Encuesta de satisfacción general ......................................................................29 


\section{INTRODUCCIÓN}

Según la Superintendencia Nacional de Educación Superior Universitaria (SUNEDU, 2018), actualmente existen 115 universidades en Perú, de las cuales 56 se encuentran en el departamento de Lima. Del total de universidades que se encuentran en Lima, solo 31 de ellas han obtenido el licenciamiento (55.4\%). Considerando solo las 31 universidades licenciadas en Lima, existen aproximadamente 250 carreras ofrecidas en el mercado de educación superior. Si a ello se le suman las carreras de las universidades no licenciadas, así como las de los institutos, el número total es aún mayor.

Además de la cantidad considerable de opciones disponibles para una tomar una decisión vocacional, se debe tomar en consideración que la edad promedio de egreso de los colegios en el Perú es de 16.7 años (Ministerio de Educación [MINEDU], 2011). Según Ginzberg, a esa edad el adolescente se encuentra en el período tentativo de su desarrollo vocacional, donde toma sus decisiones en base a sus intereses, capacidades y valores (como se citó en López, 2013).

Por ello, se considera importante contar con programas de orientación vocacional en la Educación Básica Regular (EBR), donde se guíe al alumno en el reconocimiento de sus habilidades e intereses y se brinde información pertinente para que tome una decisión adecuada.

En el presente trabajo, se describirá el Programa de Orientación Pre Vocacional que utiliza una institución educativa pública en Lima Metropolitana dirigido a alumnos del cuarto año de secundaria. En los últimos capítulos, se procederá a discutir los resultados del programa, así como a analizar los logros y las oportunidades de mejora del mismo. 


\section{CAPÍTULO I: IDENTIFICACIÓN DEL PROBLEMA}

La definición conceptual, identificación de alcances y determinación del rol de la orientación vocacional ha cambiado con el tiempo. Pássera (2014) explica que, entre los años '50 y '70, esta dejó de tener un papel directivo (imposición de carrera) para ser un proceso de guía que toma en cuenta las aptitudes y preferencias personales.

Este cambio conllevó a que se adopte un enfoque integral, considerando los aspectos evolutivos, psicodinámicos y psicosociales del individuo (López, 2013). Es decir, se da importancia a la trayectoria de la persona, en qué momento de su desarrollo se encuentra, sus motivaciones, habilidades, perspectivas y entorno social.

Planas, Cobos y Gutiérrez-Crespo (2012) definen la orientación vocacional como un conjunto de actividades realizadas en el ámbito educativo cuya finalidad es que los alumnos reconozcan sus capacidades e intereses, así como comunicarles las opciones existentes en la educación superior y la realidad laboral actual.

Algunas ventajas de recibir orientación vocacional en la escolaridad son:

- Permite que el individuo descubra habilidades que desconocía como propias, lo cual mejora su autoconcepto y autoeficacia.

- Ayuda a resolver las dudas que el estudiante tenga sobre los datos específícos de una carrera, tales como la duración, la currícula, los campos de trabajo, entre otros.

- Facilita la construcción del plan de vida, ya que permite fijarse un objetivo.

- Guía en la toma de decisiones, considerando la información recogida y el contexto individual (Ministerio de Trabajo y Promoción del Empleo del Perú [MTPE], 2015). 
Además de lo expuesto, existen también importantes riesgos a falta de una orientación vocacional. Uno de ellos es la deserción en el sistema de educación superior. Según Castaño, Gallón, Gómez y Vásquez, la deserción involucra cambiarse de centro de estudios y/o de carrera, y puede darse desde que el alumno cuenta con un cupo, mas no se matricula, hasta que se retira en el último ciclo de la carrera (como se citó en Viale, 2014, p. 61). En el Perú, se ha documentado la deserción en la educación superior a causa de razones vocacionales.

Heredia, et al. (2015) analizaron datos de todos los estudiantes egresados en el 2010 de carreras relacionadas a medicina en nueve universidades peruanas. Encontraron que el aspecto vocacional fue el principal motivo reportado por el $50 \%$ de los encuestados que desertaron.

Según el Diario Gestión (2017), el 27\% de los ingresantes a universidades privadas del Perú abandonó su carrera en los dos primeros ciclos. Este porcentaje se elevó al $48 \%$ al considerar a quienes permanecieron más de dos ciclos, mas no culminaron la carrera. Reportaron que, entre los principales factores de deserción, se encontraban las dudas vocacionales.

Bobadilla (2018) encuestó a 359 estudiantes de Estudios Generales Letras de la Pontificia Universidad Católica del Perú. Entre los desertores, el 20\% dio como motivo que no les gustó la carrera, siendo la causa con mayor porcentaje. Asimismo, 66.8\% de quienes estaban considerando cambiarse de carrera lo harían porque la actual no era afín a su vocación.

Estas investigaciones sugieren el grave impacto que una orientación vocacional ineficiente puede tener académica, laboral y económicamente (Steren, Arriaga y Costa, 2013). A pesar de la relevancia de la orientación vocacional, existen pocas investigaciones sobre los factores que influyen en su ineficiencia. No obstante, existen algunos factores atribuidos al fracaso de este proceso. Entre ellos se encuentra la falta de instrumentos psicológicos adecuados, un 
departamento de psicología poco articulado o inexistente y la transmisión de información incompleta sobre la oferta de opciones vocacionales ofrecida por parte del centro educativo.

Esta problemática demuestra una necesidad en el ámbito educativo, ya que, al mejorar los factores mencionados durante la EBR, puede disminuir el riesgo de deserción. Esto puede lograrse si las escuelas brindan información pertinente de la educación superior y guían al alumno en la detección de sus habilidades y motivaciones. Es relevante hacerlo en la etapa escolar, ya que el $82 \%$ de las personas inician su interés vocacional entre secundaria y la universidad (Bobadilla, 2018).

Esta misma necesidad se encontró en un colegio público ubicado en la ciudad de Lima. Cuenta con los niveles de Inicial, Primaria y Secundaria, así como ofrece el programa de Bachillerato Internacional para alumnos de cuarto y quinto de secundaria. Tiene una población total aproximada de 2000 estudiantes.

La primera implementación de un programa de orientación vocacional en dicha institución se dio en 1988. Luego de encuestar a egresados de 5 años previos, se halló que el 14\% de los participantes había desertado durante su trayecto universitario por una baja conformidad con su elección.

En base a esto, la institución tomó la decisión de desarrollar el Programa de Orientación Vocacional (POV) para los alumnos de quinto de secundaria, así como un Programa de Orientación Pre Vocacional (POPV), dirigido a los de cuarto. Inicialmente, ambos programas consistían únicamente en la aplicación de un test psicológico para luego entregar un informe al alumno. A partir del 2008, adicionalmente, se implementaron las ferias vocacionales, se distribuyó información de las universidades e institutos, y se mejoró el proceso de evaluación. En los siguientes capítulos, se revisarán las actividades y resultados del POPV. 


\section{CAPÍTULO II: DESCRIPCIÓN DE LAS ACTIVIDADES Y TAREAS REALIZADAS}

El POPV 2018 tuvo como objetivo orientar a los alumnos de cuarto de secundaria durante su proceso de identificación vocacional. Para lograr esto, se dividió en cuatro fases.

\subsection{Fase de promoción}

El objetivo de esta fase fue que el alumno conozca la oferta existente en el mercado de la educación superior. La actividad final de esta fase fue la feria vocacional, para la cual se consideraron las siguientes etapas.

\subsubsection{Etapa de planificación}

Se escogieron las universidades e institutos a convocar para coordinar la fecha, horario y sus requerimientos para la feria. Las instituciones se seleccionaron a partir de encuestas realizadas en años anteriores. Entre las funciones desarrolladas, se sugirió considerar como requisito que cuenten con licenciamiento institucional. Esto garantizó que los invitados cumplieran con las Condiciones Básicas de Calidad (SUNEDU, 2016).

Se convocó un total de 17 instituciones, entre universidades e institutos, tanto públicas como privadas. Se apoyó a la institución coordinando con tres del total escogido.

Se estableció que la feria vocacional fuera el primer sábado de mayo, para disponer de dos meses de coordinación, así como para facilitar la asistencia de padres de familia y alumnos por ser fin de semana. 


\subsubsection{Etapa de desarrollo}

Cada psicóloga ingresó a las aulas de secundaria para entregar los comunicados informativos sobre la feria vocacional a los alumnos. También se motivó su asistencia a la misma, reflexionando con los alumnos sobre su utilidad e importancia. La responsabilidad asignada consistió en ingresar a siete aulas de tercero de secundaria (187 alumnos) para brindar detalles de la feria e incentivar su participación.

\subsubsection{Etapa final}

Se llevó a cabo la feria vocacional de 9:00am a 12:00pm, en el patio central, siendo el espacio con mayor capacidad de aforo.

Se apoyó en la feria ubicando a los representantes de las tres universidades asignadas y asegurando que tuvieran los requerimientos solicitados (puntos de luz, mesas, sillas, etc.), así como dando la bienvenida a los asistentes y guiándolos a través de la feria.

\subsection{Fase de detección}

El objetivo de esta fase fue identificar las habilidades e intereses de los alumnos de cuarto de secundaria, para luego comunicarlas en una sesión de orientación con alumnos y padres. Para llegar a ello, se desarrollaron las siguientes actividades.

\subsubsection{Etapa de planificación}

En esta etapa se planificó la evaluación de las aptitudes e intereses de los estudiantes a través de pruebas psicológicas. También se coordinó con las autoridades las fechas y horas en las cuales se evaluaría a los alumnos.

Se escogió evaluar la inteligencia, pues es "una parte esencial en la evaluación psicopedagógica, ya que permite diseñar recomendaciones 
personalizadas y ajustadas durante las labores de orientación vocacional..." (Sánchez-Sánchez, 2015, p. 58). Asimismo, se decidió evaluar los intereses de los estudiantes respecto a sus posibilidades vocacionales.

La institución utilizaba el Inventario de Preferencias Profesionales (De la Cruz, 1993). Como aporte al programa, se sugirió que se aplicara el Inventario de Preferencias Profesionales Revisado (De la Cruz, 2010), ya que esta versión fue recientemente actualizada y adaptada a una muestra limeña por Olivera, Uribe y Denegri (2013).

Para evaluar la inteligencia, se aplicaba la Batería de Evaluación de la Inteligencia General y Factorial - Revisada (Yuste, 2002). No obstante, no se encontraron investigaciones que evidencien su adecuado funcionamiento psicométrico en Lima. Por ello, como segundo aporte, se propuso reemplazar el IGF-r5 por la prueba Sternberg Triarchic Abilities Test, Nivel H ([STAT], Sternberg, 1991), ya que es una prueba de inteligencia de aplicación colectiva que fue traducida y adaptada por Carrasco (2000) y cuenta con propiedades psicométricas adecuadas.

Se consideró importante utilizar pruebas con propiedades psicométricas adecuadas para asegurar que las decisiones que se tomen sobre ellas sean acertadas. (Olivera, et al., 2013).

\subsubsection{Etapa de desarrollo}

Para la evaluación, se envió un formato de Consentimiento Informado a los padres de familia con la finalidad de contar con su autorización para que sus hijos puedan ser evaluados como parte del POPV. La promoción de cuarto de secundaria estaba conformada por 192 alumnos, distribuidos en cuatro aulas de Secundaria Regular y tres de Bachillerato. De ese total, se 
obtuvo el consentimiento de 188 estudiantes, pues cuatro no accedieron. Se tuvo la responsabilidad de entregar y recoger los consentimientos de dos aulas de cuarto de secundaria (56 alumnos). Esta etapa se llevó a cabo según el cronograma que se elaboró para su ejecución (ver Apéndice 1).

Para el proceso de evaluación, cada psicóloga fue asignada a un aula. El primer día se explicó dicho proceso a los alumnos, así como el valor que aporta cada prueba y la importancia de desarrollarlas de forma honesta y seria. Esto se realizó en los primeros 15 minutos del día, correspondientes a tutoría. Luego, se aplicó en todas las aulas el test STAT en la primera hora pedagógica, dando un total de 45 minutos para el desarrollo de la prueba. Los estudiantes comprendieron las indicaciones y culminaron en el tiempo designado.

Al día siguiente se aplicó el IPP-R, repitiendo el horario del día anterior. Las siete aulas desarrollaron a la vez el test, completándolo dentro de los 45 minutos disponibles. Los días restantes de esa semana fueron designados para evaluar a aquellos alumnos que no asistieron durante los días previos. Las aulas contaron con iluminación y ventilación adecuada. También estuvieron libres de distracciones.

El siguiente paso fue la corrección de las pruebas aplicadas. Cada psicóloga estuvo a cargo de corregir las pruebas de 32 alumnos y redactar los informes respectivos. Se utilizaron los manuales respectivos de cada prueba para su corrección y se procedió a redactar los informes en base al formato elaborado por la institución (ver Apéndice 3). 


\subsubsection{Etapa final}

Se programaron sesiones de asesoría individual con cada alumno evaluado, durante las cuales se revisaron sus informes y se aclararon sus dudas. Luego, se citó a los padres de cada estudiante para hacer entrega del informe, brindar pautas para apoyar a sus hijos durante el proceso de decisión vocacional y resolver preguntas al respecto. La responsabilidad asignada consistió en citar a 32 alumnos y sus padres.

\subsection{Fase de exposición}

El objetivo de esta fase fue presentar las experiencias de estudiantes egresados a los alumnos. Se buscó que los graduados compartieran su proceso vocacional, así como su trayectoria educativa y profesional. Para ello, se ofreció a los estudiantes una charla dirigida por los alumnos egresados. Esto fue añadido al POPV 2018 por sugerencia a la institución como un aporte al programa.

\subsubsection{Etapa de planificación}

Como etapa inicial, se coordinó con el departamento de Tutoría y Orientación Educativa que asistiera un egresado por aula. Por ello, la institución asignó la responsabilidad de contactar de forma personal a 30 exalumnos.

Se logró contactar la cantidad solicitada de exalumnos, la cual representaba una diversidad de edades y tipos de carrera. La fecha final acordada fue el lunes 27 de agosto de 2018, durante las dos primeras horas pedagógicas.

\subsubsection{Etapa de desarrollo}

Se preparó una sesión con los exalumnos con la finalidad de exponer el objetivo de la charla que darían. Se tuvo la responsabilidad de elaborar el 
material de exposición y dirigir una sesión de 20 minutos. Se les solicitó compartir su experiencia sobre el proceso de decisión vocacional. Esto incluyó considerar por qué estudiaron la carrera escogida en la institución a la que asistieron, así como qué aprendizajes tuvieron en el transcurso de su educación superior y eventual experiencia laboral. Además, se brindaron pautas sobre el manejo de grupo.

\subsubsection{Etapa final}

Se contó con la asistencia de todos los exalumnos invitados, por lo cual todas las aulas de secundaria participaron de la actividad.

Cada uno ingresó a un salón y compartió sus experiencias durante aproximadamente 45 minutos, utilizando los 15 minutos restantes para resolver las preguntas de los estudiantes. Como parte de las tareas realizadas, se brindó apoyo en el manejo de grupo en un aula de tercero.

\subsection{Fase de información continua}

El objetivo de esta fase fue informar a los alumnos a través de un panel vocacional ubicado en el patio de secundaria. En él, se exponía la información que enviaban las universidades e institutos sobre las carreras, requisitos, modalidades de admisión y fechas importantes. Como aporte al programa, se propuso añadir las fechas de los simulacros de exámenes de admisión, ya que dan posibilidades de práctica a los estudiantes. Esto ayuda a reducir los niveles de ansiedad y temor que se suelen presentar frente a las evaluaciones (Mayo, González-Freire y Trillo, 2015). 


\section{CAPÍTULO III: RESULTADOS DE LA INTERVENCIÓN}

En el presente capítulo se discuten los resultados obtenidos del POPV, tanto los de cada fase, como del programa en general.

\subsection{Resultados de la fase de promoción}

La feria vocacional contó con la asistencia de 14 universidades y dos institutos. En total, constituyen un $26 \%$ del total de instituciones licenciadas disponibles en el mercado de educación superior en Lima (SUNEDU, 2018; MINEDU, 2019). Comparativamente, esta feria contó con el nivel más alto de participación de la que se tenga registro en la institución.

Durante la fase de promoción, se estimó la asistencia de al menos 216 personas. Esta cifra fue ampliamente superada, pues se contabilizaron 245 participantes a partir del conteo de encuestas entregadas. Esta cifra representó un incremento del $39 \%$ en comparación a la asistencia registrada en el 2017.

Otro logro fue la satisfacción de los asistentes respecto a la feria. El 98\% manifestó estar "muy satisfecho" y "bastante satisfecho" con la organización De igual forma, un $63 \%$ de los asistentes estuvo "bastante satisfecho" con la utilidad de la información presentada.

La sugerencia realizada dentro de la presente fase fue aceptada con facilidad por el centro laboral, pues reconocieron el valor de informar a los alumnos únicamente sobre instituciones con licenciamiento.

\subsection{Resultados de la fase de detección}

Se cumplió con la redacción del 100\% de los informes de orientación pre vocacional dentro del tiempo establecido. Además, se brindó la sesión de orientación individual al 100\% de los alumnos evaluados. 
Lo mencionado anteriormente está alineado con el objetivo de la fase de detección. Es decir, se guió al alumno en el reconocimiento de sus intereses y habilidades a través de las evaluaciones completadas y de las sesiones de orientación desarrolladas.

Hubo dificultades al implementar las sesiones de orientación con los padres. De las 188 familias citadas, solo asistieron 33 (18\%). Esto impide que los padres revisen el informe con las psicólogas y que absuelvan sus dudas al respecto. Una posible consecuencia es que los padres se comuniquen con sus hijos de una manera poco asertiva e informada sobre su futuro vocacional.

Se sugirió a la institución reemplazar las pruebas psicológicas en uso por otras con adecuadas propiedades psicométricas en el contexto limeño. En un principio, hubo dificultades en la aceptación de la recomendación. Se argumentó que las pruebas utilizadas no habían generado inconvenientes. Frente a ello, se reiteró la importancia de tener la certeza de que los resultados obtenidos reflejen la realidad. Finalmente, se aceptó la propuesta y las pruebas mencionadas se incorporaron en el POPV en lugar de las utilizadas previamente.

\subsection{Resultados de la fase de exposición}

La charla de intercambio de experiencias contó con la participación de aproximadamente 840 alumnos. Un logro obtenido fue el nivel de satisfacción de los alumnos frente a la charla. Un $64.2 \%$ de los estudiantes consideró que la charla fue de gran utilidad y un $82 \%$ estuvo de acuerdo con repetir la actividad en el 2019.

Un indicador más de la adecuada planificación de esta fase fue el interés de los estudiantes durante la charla, el cual se reflejó en las preguntas dirigidas a los 
expositores. La fase de exposición fue una propuesta realizada al colegio que fue aceptada con facilidad por las autoridades.

\subsection{Resultados de la fase de información continua}

Para medir el impacto del panel vocacional, se encuestó a los alumnos de cuarto de secundaria. El 69\% refirió consultar la información colocada voluntariamente, se encontró satisfecho con los datos exhibidos y lo percibió como útil.

La sugerencia de incorporar las fechas de exámenes simulacro al panel fue aceptada sin dificultades.

\subsection{Resultados del Programa de Orientación Pre Vocacional}

En las encuestas de satisfacción sobre el POPV, un $83 \%$ estuvo "totalmente de acuerdo" con que participar en las actividades del programa ayudó a conocer sus opciones vocacionales y tomar una decisión informada.

Además, los índices de satisfacción de cada fase del programa superaron el $60 \%$. Por ello, el programa cumplió con el objetivo de orientar a los alumnos de cuarto de secundaria durante su proceso de descubrimiento vocacional. Este logro fue posible gracias a la combinación de actividades informativas, de evaluación y de consejería que formaron parte del programa. Estas herramientas son necesarias dentro del proceso orientador (Vargas, 2018). Sin embargo, aún no se evalúa el impacto del POPV a largo plazo. Para ello, se necesita hacer seguimiento a la tasa de deserción en los alumnos egresados que hayan participado del programa.

Ofrecer una orientación vocacional en las escuelas facilita que los alumnos basen su elección en información actual y relevante de la educación superior, el mercado laboral y de sus fortalezas e intereses. El POPV de la institución representa un aporte aplicativo, ya que ha demostrado que contar con distintas actividades informativas ayuda al alumno a tomar una decisión vocacional mejor sustentada. 


\section{CONCLUSIONES}

- El POPV permite la detección de las habilidades y preferencias de los alumnos, así como informa de forma pertinente sobre la oferta de la educación superior y el contexto laboral.

- Es necesario hacer seguimiento para evaluar el impacto del programa a largo plazo.

- Los alumnos y padres de familia encontraron valor en la información que encontraron en la feria vocacional de la institución.

- Una mayor oferta de instituciones de educación superior en la feria vocacional genera mayor interés y acogida por parte de sus participantes.

- Los padres de familia presentan bajo interés en los informes psicológicos vocacionales, lo cual dificulta la guía en casa.

- El panel vocacional facilita que los alumnos encuentren información relevante sobre universidades e institutos.

- El intercambio de experiencias con exalumnos permite que los estudiantes conozcan de forma detallada el proceso vocacional y la etapa posterior al colegio. 


\section{RECOMENDACIONES}

- Hacer seguimiento a las promociones egresadas a través de encuestas para evaluar el impacto del POPV a largo plazo y adaptarlo en base a la información encontrada.

- Realizar una charla con los padres de familia para explicar el objetivo e importancia del POPV, específicamente de la fase de detección, para así lograr el 100\% de participación en las evaluaciones y en las sesiones de orientación individual.

- Mantener o aumentar la cantidad de instituciones de educación superior convocadas para la feria vocacional, ya que esto motiva la asistencia de la comunidad escolar.

- Continuar utilizando el panel vocacional como medio informativo alternativo para los alumnos sobre eventos y fechas importantes.

- Realizar encuestas para detectar las carreras de mayor interés entre los estudiantes y así convocar a los exalumnos que las estudien o hayan estudiado; esto permite que los alumnos reciban información más personalizada.

- En base a las mismas encuestas, identificar las carreras mencionadas con menor frecuencia y preparar charlas de asistencia voluntaria para atender los intereses minoritarios.

- Involucrar a los docentes y tutores como parte del programa, ya que pueden brindar más información sobre las aptitudes que detectan en sus estudiantes. 


\section{REFERENCIAS}

Bobadilla, P. (2018). ¿Y...Cómo eliges tu profesión? Motivaciones y factores que influyen en la elección de una carrera profesional en estudiantes de estudios generales letras y sociología de la PUCP. Lima: Departamento de Ciencias Sociales, PUCP. Recuperado de http://repositorio.pucp.edu.pe/index/handle/123456789/112449

Carrasco, M. P. (2000). Adaptación, validez y confiabilidad del Sternberg Triarchic Abilities Test - Modified Nivel H en estudiantes preuniversitarios de estudios generales de una universidad privada de Lima (tesis para optar el título profesional de Licenciado en Psicología). Universidad de Lima.

De la Cruz, M. V. (1993). IPP: Inventario de Intereses y Preferencias Profesionales. Madrid: TEA Ediciones.

De la Cruz, M. V. (2010). Intereses y Preferencias Profesionales (3. ${ }^{\mathrm{a}}$ ed.). Madrid: TEA Ediciones.

Gestión (11 de julio de 2017). El 27\% de ingresantes a universidades privadas abandonan su carrera en primer año de estudios. Diario Gestión. Recuperado de https://gestion.pe/tendencias/management-empleo/27-ingresantes-universidadesprivadas-abandonan-carrera-primer-ano-estudios-139168

Heredia, M., Andía, M., Ocampo, H., Ramos-Castillo, J., Rodríguez, A., Tenorio, C., y Pardo, K. (2015). Deserción estudiantil en las carreras de ciencias de la salud en el Perú. Anales de la Facultad de Medicina, 76, 57-61. doi: http://dx.doi.org/10.15381/anales.v76i1.10972 
López Bonelli, A. R. (2013). La orientación vocacional como proceso: teoría, técnica y práctica (4. ${ }^{\mathrm{a}}$ ed.). Buenos Aires: Bonum.

Mayo, E., González-Freire, B. y Trillo, V. (2015). Ansiedad ante los exámenes en la universidad: estudio de caso único. Ansiedad y estrés, 21(1), 21-33. Recuperado de http://www.ebsco.com

Ministerio de Educación del Perú (enero de 2011). Segundo Censo Nacional Universitario 2010.

Recuperado

de

http://repositorio.minedu.gob.pe/bitstream/handle/123456789/865/503.\%20II\%20Cen so $\% 20$ Nacional $\% 20$ Universitario\%202010\%20Principales\%20resultados.pdf?sequen ce $=1 \&$ is Allowed $=\mathrm{y}$

Ministerio de Educación del Perú (04 de febrero de 2019). Listado de IES licenciados en el año 2018. Recuperado de http://www.minedu.gob.pe/ley-de-institutos/pdf/ieslicenciados-final.pdf

Ministerio de Trabajo y Promoción del Empleo del Perú (04 de diciembre de 2015). La importancia de la orientación vocacional. Recuperado de http://www.trabajo.gob.pe/archivos/file/dnpefp/SOVIO/articulos_interes/ARTICULO _DE_INTERES_1.pdf

Olivera, C., Uribe, P. y Denegri, N. (2013). Validación del IPP-R con alumnos de cuarto y quinto grado de secundaria de colegios públicos y privados de Lima metropolitana. Persona, 16, 139-164. Recuperado de http://revistas.ulima.edu.pe/index.php/Persona/article/view/7/7

Pássera, J. (2014). Orientación vocacional: Una propuesta teórico-práctica (1. ${ }^{\text {a }}$ ed.). Córdoba: Editorial Brujas.

Planas, J., Cobos, A. y Gutiérrez-Crespo, E. (2012). Manual de asesoramiento y orientación vocacional (1. ${ }^{\mathrm{a}}$ ed.). Madrid: Editorial Síntesis. 
Sánchez-Sánchez, F. (2015). La evaluación inteligente de la inteligencia. Padres y Maestros, (364), 55-63. doi: https://doi.org/10.14422/pym.i364.y2015.009

Steren, B., Arriaga, J. y Costa, M. (2013). Una visión integral del abandono. Porto Alegre, Brasil: EDIPUCRS.

Recuperado de http://ridda2.utp.ac.pa/bitstream/handle/123456789/3143/Una-Vision-Integral-delAbandono.pdf? sequence $=1 \&$ isAllowed $=\mathrm{y}$

Sternberg, R. J. (1991). Theory-based testing of intellectual abilities: Rationale for the triarchic abilities test. En H.A. Rowe (Ed.), Intelligence: Reconceptualization and measurement (pp. 183-202). Hillsdale: Lawrence Erlbaum Associates.

Superintendencia Nacional de Educación Superior Universitaria (15 de enero de 2018). Lista de universidades peruanas. Recuperado de https://www.sunedu.gob.pe/listauniversidades/

Superintendencia Nacional de Educación Superior Universitaria (enero de 2016). El Modelo de Licenciamiento y su Implementación en el Sistema Universitario Peruano. Recuperado de https://www.sunedu.gob.pe/wpcontent/uploads/2016/06/modelo_licenciamiento_institucional.pdf

Vargas, M. A. (2018) La inforgrafía como herramienta útil para dar a conocer la mala elección de una carrera universitaria, debido a la ausencia de orientación vocacional en adolescentes (tesis para optar el título profesional de Licenciado en Arte y Diseño Empresarial). Universidad San Ignacio de Loyola.

Viale, H. (2014). Una aproximación teórica a la deserción estudiantil universitaria. Revista Digital de Investigación en Docencia Universitaria, 8(1), 59-76. doi: https://doi.org/10.19083/ridu.8.366

Yuste, C. (2002). Inteligencia General Factorial - Revisada 5 (IGF-r5). Madrid: EOS. 


\section{APÉNDICES}




\section{APÉNDICE 1: PLANIFICACIÓN DEL PROGRAMA DE}

\section{ORIENTACIÓN PRE VOCACIONAL}

\begin{tabular}{|c|c|c|c|c|c|c|c|c|}
\hline \multicolumn{9}{|c|}{ PROGRAMA DE ORIENTACIÓN PRE VOCACIONAL - 4TO SECUNDARIA 2018} \\
\hline & 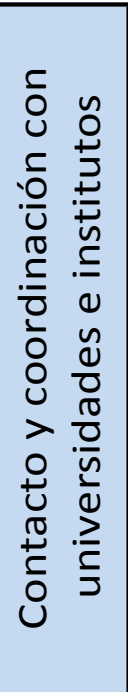 & 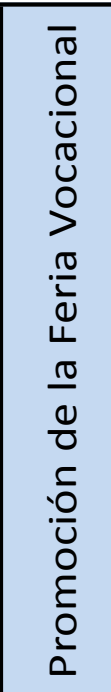 & 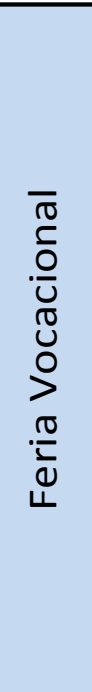 & 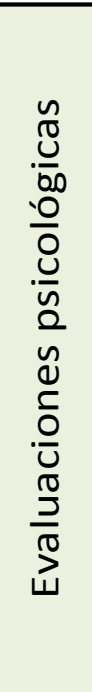 & 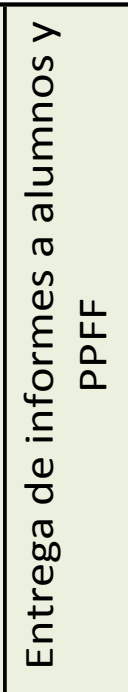 & 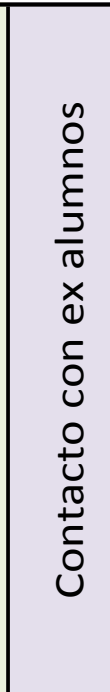 & 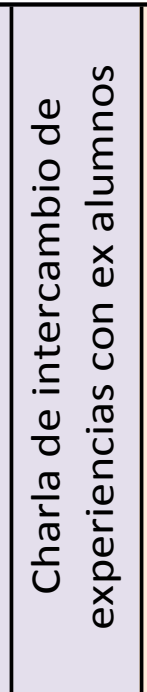 & 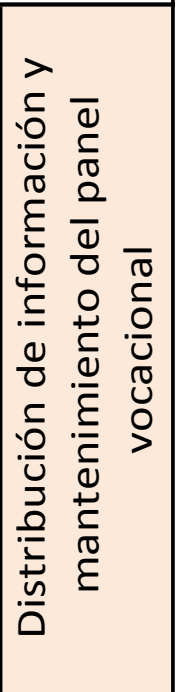 \\
\hline \multicolumn{9}{|l|}{ Marzo } \\
\hline \multicolumn{9}{|l|}{ Abril } \\
\hline \multicolumn{9}{|l|}{ Mayo } \\
\hline \multicolumn{9}{|l|}{ Junio } \\
\hline \multicolumn{9}{|l|}{ Julio } \\
\hline \multicolumn{9}{|l|}{ Agosto } \\
\hline \multicolumn{9}{|l|}{ Septiembre } \\
\hline \multicolumn{9}{|l|}{ Octubre } \\
\hline \multicolumn{9}{|l|}{ Noviembre } \\
\hline \multicolumn{9}{|l|}{ Diciembre } \\
\hline & & Fase d & Promo & & & Fase o & Exposici & \\
\hline & & Fase d & Deteco & & & Fase c & Informa & ción Continu \\
\hline
\end{tabular}




\section{APÉNDICE 2: RESUMEN DE TAREAS REALIZADAS}

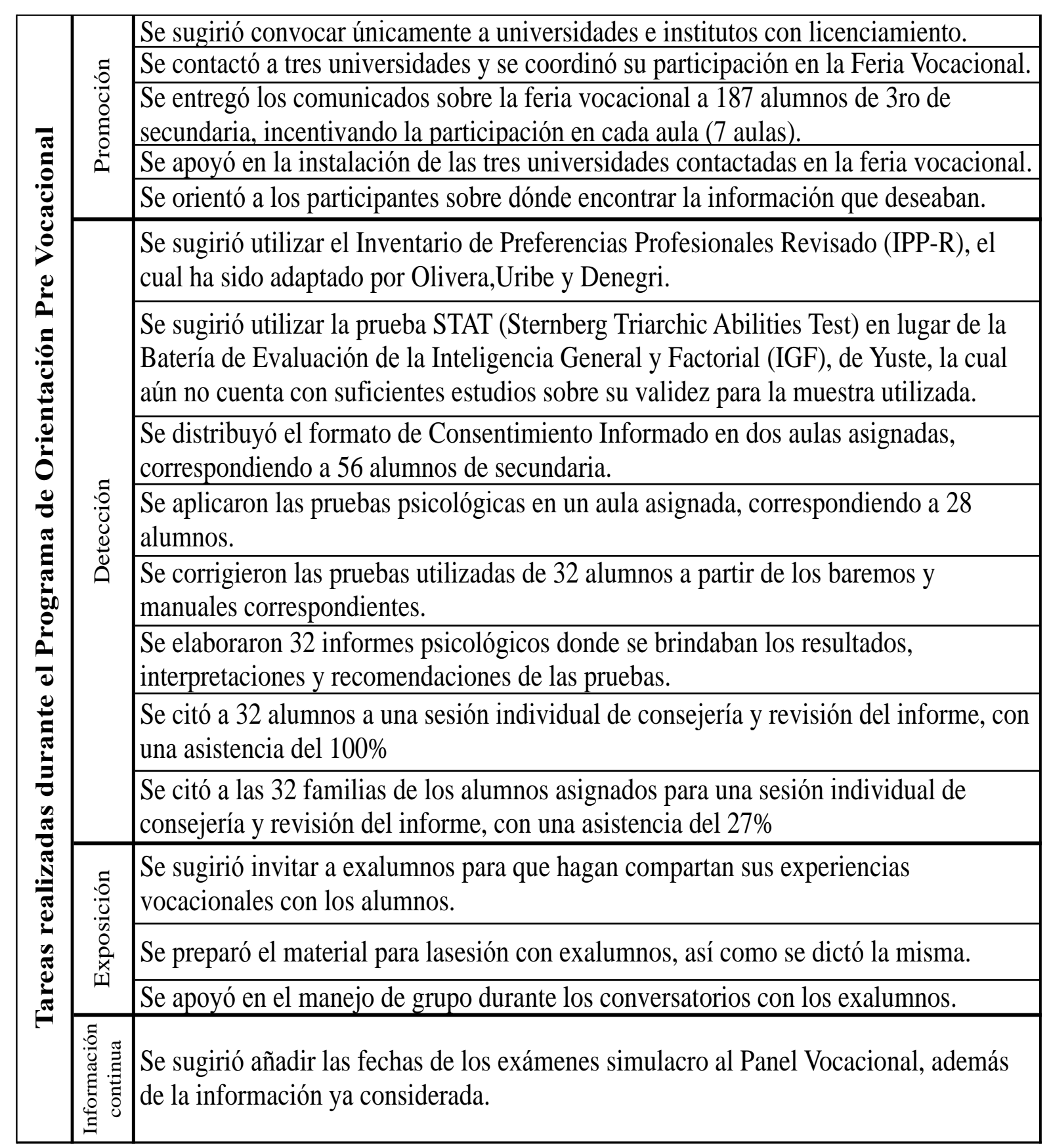




\section{APÉNDICE 3: MODELO DE INFORME VOCACIONAL}

INFORME PRE VOCACIONAL

\section{DATOS PERSONALES}

Apellidos y Nombres:

Edad:

Fecha de Nacimiento:

C. Educativo:

Grado y sección:

Fecha de Informe:

\section{MOTIVO DE INFORME}

Evaluación al alumno(a) como parte del Programa de Orientación Pre Vocacional.

\section{OBSERVACIONES GENERALES}

\section{METODOLOGÍA}

- Aplicación de pruebas psicológicas:

- STAT (Inteligencia)

○ IPP-R (intereses)

\section{RESULTADOS DE LAS PRUEBAS PSICOLÓGICAS APLICADAS}

- Área Intelectual

- Intereses

\section{CONCLUSIONES}

RECOMENDACIONES 


\section{APÉNDICE 4: ENCUESTAS DE SATISFACCIÓN PARA}

\section{ALUMNOS DE 4 DE SECUNDARIA}

\begin{tabular}{|r|l|c|c|c|c|c}
\hline & $\begin{array}{l}\text { Marca del } 1 \text { al } 5 \text { en base a qué tan satisfecho(a) te sentiste } \\
\text { respecto a: }\end{array}$ & $\begin{array}{c}1 \\
\text { (Muy } \\
\text { Satisfecho) }\end{array}$ & $\begin{array}{c}2 \\
\text { (Bastante } \\
\text { satisfecho }\end{array}$ & $\begin{array}{c}3 \\
\text { (Indiferente) }\end{array}$ & $\begin{array}{c}4 \\
\text { (Poco } \\
\text { satisfecho) }\end{array}$ & $\begin{array}{c}5 \\
\text { (Nada } \\
\text { satisfecho) }\end{array}$ \\
\hline 1 & La Feria Vocacional 2018, en general & & & & & \\
\hline 2 & El horario escogido (de 9:00am a 12:00pm) & & & & & \\
\hline 3 & El día escogido (sábado) & & & & & \\
\hline 4 & $\begin{array}{l}\text { La oferta de universidades e institutos que pudo } \\
\text { encontrar en la Feria Vocacional }\end{array}$ & & & & & \\
\hline 5 & $\begin{array}{l}\text { La utilidad de la información sobre las carreras que } \\
\text { encontró en la Feria Vocacional }\end{array}$ & & & & & \\
\hline 6 & Comentarios adicionales: & & & & \\
\hline
\end{tabular}

\begin{tabular}{|c|c|c|c|c|c|c|}
\hline & $\begin{array}{l}\text { Lee las siguientes preguntas y marca del } 1 \text { al } 5 \text { según tu } \\
\text { concordancia respecto a los siguientes enunciados: }\end{array}$ & $\begin{array}{l}1 \\
\text { (Totalment } \\
\text { e de } \\
\text { acuerdo) }\end{array}$ & $\begin{array}{c}2 \\
\text { (De } \\
\text { acuerdo) }\end{array}$ & $\begin{array}{c}3 \\
\text { (Indiferente) }\end{array}$ & $\begin{array}{c}4 \\
(\text { En } \\
\text { desacuerdo } \\
)\end{array}$ & $\begin{array}{c}5 \\
\text { (Totalmente } \\
\text { en } \\
\text { desacuerdo) }\end{array}$ \\
\hline 1 & $\begin{array}{l}\text { La información que recibí en la charla con } \\
\text { exalumnos fue útil para mis decisiones } \\
\text { vocacionales. }\end{array}$ & & & & & \\
\hline 2 & $\begin{array}{l}\text { La persona que habló en mi salón había estudiado } \\
\text { algo relacionado a mis intereses. }\end{array}$ & & & & & \\
\hline 3 & Se debería repetir la charla en los siguientes años. & & & & & \\
\hline 4 & $\begin{array}{l}\text { Si realicé preguntas, fueron contestadas de forma } \\
\text { clara. }\end{array}$ & & & & & \\
\hline 5 & $\begin{array}{l}\text { La charla me ayudó a comprender sobre el proceso } \\
\text { de elección de una carrera y lugar para estudiar. }\end{array}$ & & & & & \\
\hline 6 & $\begin{array}{l}\text { Si tenía preocupaciones sobre mi futuro vocacional, } \\
\text { esta charla ayudó a aclarar mis dudas y sentirme } \\
\text { más tranquilo(a). }\end{array}$ & & & & & \\
\hline
\end{tabular}

\begin{tabular}{|r|l|c|c|c|c|c}
\hline \multicolumn{2}{|l}{ ENCUESTA - PANEL VOCACIONAL } \\
$\begin{array}{l}\text { Lee las siguientes preguntas y marca del 1 al 5 según tu } \\
\text { concordancia respecto a los siguientes enunciados: }\end{array}$ & $\begin{array}{c}1 \\
\text { (Totalment } \\
\text { e de } \\
\text { acuerdo) }\end{array}$ & $\begin{array}{c}2 \\
\text { (De } \\
\text { acuerdo) }\end{array}$ & $\begin{array}{c}3 \\
\text { (Indiferente) }\end{array}$ & $\begin{array}{c}4 \\
\text { (En } \\
\text { desacuerdo } \\
\text { ) }\end{array}$ & $\begin{array}{c}\text { (Totalmente } \\
\text { en } \\
\text { desacuerdo) }\end{array}$ \\
\hline 2 & $\begin{array}{l}\text { A lo largo del año, me he acercado por interés } \\
\text { propio al Panel Vocacional para consultar } \\
\text { información. }\end{array}$ & $\begin{array}{l}\text { La información que encontré en el Panel Vocacional } \\
\text { ha sido de mi interés. }\end{array}$ & & & & \\
\hline 3 & $\begin{array}{l}\text { La información que encontré en el Panel Vocacional } \\
\text { fue útil para resolver mis dudas. }\end{array}$ & & & & & \\
\hline 4 & $\begin{array}{l}\text { Considero que el Panel Vocacional debe volver a } \\
\text { utilizarse en los siguientes años. }\end{array}$ & & & & & \\
\hline
\end{tabular}




\section{APÉNDICE 5: ENCUESTA DE SATISFACCIÓN GENERAL}

\section{PARA ALUMNOS DE $4^{\circ}$ DE SECUNDARIA}

ENCUESTA - ORIENTACIÓN VOCACIONAL

\begin{tabular}{|l|l|l|l|l|l|c|}
\hline & $\begin{array}{l}\text { Lee las siguientes preguntas y marca del 1 al } \\
5 \text { según tu concordancia respecto a los } \\
\text { siguientes enunciados: }\end{array}$ & $\begin{array}{c}1 \\
\text { (Totalmente } \\
\text { de acuerdo) }\end{array}$ & $\begin{array}{c}2 \\
\text { (De acuerdo) }\end{array}$ & $\begin{array}{c}3 \\
\text { (Indiferente) }\end{array}$ & $\begin{array}{c}4 \\
\text { (En desacuerdo) }\end{array}$ & $\begin{array}{c}\text { (Totalmente en } \\
\text { desacuerdo) }\end{array}$ \\
\hline $\begin{array}{l}\text { Participé en tres o más de las siguientes } \\
\text { actividades: feria vocacional, evaluación, } \\
\text { sesión de consejería individual, panel } \\
\text { vocacional y charla de intercambio de } \\
\text { experiencias con exalumnos. }\end{array}$ & & & & & \\
\hline 2 & $\begin{array}{l}\text { Por participar en las actividades } \\
\text { vocacionales, me siento más informado(a) } \\
\text { sobre las carreras, universidades e } \\
\text { institutos. }\end{array}$ & & & & & \\
\hline 3 & $\begin{array}{l}\text { Por participar en las actividades } \\
\text { vocacionales, me siento más informado(a) } \\
\text { sobre mis intereses y habilidades. }\end{array}$ & & & & & \\
\hline 4 & $\begin{array}{l}\text { Por particpar en las actividades } \\
\text { vocacionales, me siento más informado(a) } \\
\text { sobre el mercado laboral actual en relación } \\
\text { a las carreras disponibles. }\end{array}$ & & & & & \\
\hline 5 & $\begin{array}{l}\text { En general, participar de las actividades } \\
\text { vocacionales ayudó a reducir o conocer mis } \\
\text { opciones vocacionales. }\end{array}$ & & & & & \\
\hline $\begin{array}{l}\text { En general, participar de las actividades } \\
\text { vocacionales me ayudó a tener mejor } \\
\text { información para tomar una decisión } \\
\text { vocacional adecuada. }\end{array}$ & & & & & \\
\hline
\end{tabular}

